\title{
A phase I dose-escalation study of TAK-733, an investigational oral MEK inhibitor, in patients with advanced solid tumors
}

\author{
Alex A. Adjei ${ }^{1,6}$ - Patricia LoRusso ${ }^{2}$ - Antoni Ribas ${ }^{3} \cdot$ Jeffrey A. Sosman $^{4}$. \\ Anna Pavlick ${ }^{5}$ - Grace K. Dy ${ }^{6}$ Xiaofei Zhou ${ }^{7}$ - Esha Gangolli ${ }^{7,8} \cdot$ Michelle Kneissl $^{7}$. \\ Stephanie Faucette $^{7} \cdot$ Rachel Neuwirth $^{7} \cdot$ Viviana Bózon $^{7,9}$
}

Received: 19 July 2016 / Accepted: 6 September 2016 / Published online: 21 September 2016

(C) The Author(s) 2016. This article is published with open access at Springerlink.com

\begin{abstract}
Summary Purpose TAK-733, an investigational, selective, allosteric MEK1/2 inhibitor, has demonstrated antitumor effects against multiple cancer cell lines and xenograft models. This first-in-human study investigated TAK-733 in patients with solid tumors. Methods Patients received oral TAK-733 once daily on days 1-21 in 28-day treatment cycles. Adverse events (AEs) were graded using the Common Terminology Criteria for AEs version 3.0. Response was assessed using RECIST v1.1. Blood samples for TAK-733 pharmacokinetics and pharmacodynamics (inhibition of ERK phosphorylation) were collected during cycle 1. Results Fifty-one patients received TAK-733 $0.2-22 \mathrm{mg}$. Primary diagnoses included uveal melanoma (24\%), colon cancer $(22 \%)$, and cutaneous melanoma (10\%). Four patients had dose-limiting toxicities of dermatitis acneiform, plus fatigue and
\end{abstract}

Electronic supplementary material The online version of this article (doi:10.1007/s10637-016-0391-2) contains supplementary material, which is available to authorized users.

Alex A. Adjei

Adjei.Alex@Mayo.edu

1 Department of Oncology, Mayo Clinic, 200 First St, SW, Rochester, MN 55905, USA

2 Yale University, New Haven, CT, USA

3 University of California at Los Angeles Jonsson Comprehensive Cancer Center, Los Angeles, CA, USA

4 Vanderbilt-Ingram Cancer Center, Nashville, TN, USA

5 New York University Langone Medical Center, New York, NY, USA

6 Roswell Park Cancer Institute, Buffalo, NY, USA

7 Millennium Pharmaceuticals, Inc., a wholly owned subsidiary of Takeda Pharmaceutical Company Limited, Cambridge, MA, USA

8 Present address: AstraZeneca Pharmaceuticals, Waltham, MA, USA

9 Present address: Array BioPharma Inc., Boulder, CO, USA pustular rash in one patient, and stomatitis in one patient. The maximum tolerated dose was $16 \mathrm{mg}$. Common drug-related AEs included dermatitis acneiform (51 \%), diarrhea (29\%), and increased blood creatine phosphokinase $(20 \%)$; grade $\geq 3$ AEs were reported in 27 (53\%) patients. Median $\mathrm{T}_{\max }$ was $3 \mathrm{~h}$; systemic exposure increased less than dose-proportionally over the dose range $0.2-22 \mathrm{mg}$. On day 21 maximum inhibition of ERK phosphorylation in peripheral blood mononuclear cells of 46$97 \%$ was seen in patients receiving TAK-733 $\geq 8.4 \mathrm{mg}$. Among 41 response-evaluable patients, $2(5 \%)$ patients with cutaneous melanoma (one with BRAF L597R mutant melanoma) had partial responses. Conclusions TAK-733 had a generally manageable toxicity profile up to the maximum tolerated dose, and showed the anticipated pharmacodynamic effect of sustained inhibition of ERK phosphorylation. Limited antitumor activity was demonstrated. Further investigation is not currently planned.

Keywords TAK-733 $\cdot$ MEK inhibition $\cdot$ Phase $1 \cdot$ Solid tumor

\section{Introduction}

The Ras/Raf/MEK/ERK mitogen-activated cascade plays a central role in the signaling required for cell proliferation, survival, motility, differentiation, and angiogenesis [1]. Dysregulation or hyperactivation of this cascade is common in many human cancers [1, 2]. In particular, MEK is frequently activated in cancers with mutations in established upstream oncogenes [3, 4]; specifically, mutations in $R A S$ and $R A F$ oncogenes can lead to increased MEK activation [3]. The RAS gene family members include $H R A S, K R A S$, and $N R A S$, with the latter two being the isoforms commonly mutated in cancers. $R A S$ activating mutations occur in $30 \%$ of all cancers, including a high prevalence in melanoma (15-25\%) [3, 5], with $K R A S$ mutations more common in adenocarcinomas and solid 
tumors and NRAS mutations more common in leukemia, thyroid carcinoma, and malignant melanoma [6]. RAS, specifically $K R A S$ [6], is frequently mutated in colorectal cancer (CRC) and has been linked to CRC initiation and progression [7, 8]. Furthermore, approximately $8 \%$ of human tumors have mutations in $B R A F$ (a member of the $R A F$ family) - melanoma, thyroid cancer, and CRC have been associated with a high frequency of $B R A F$ mutations $[9,10]$. Specifically, the V600E point mutation accounts for more than $80 \%$ of $B R A F$ activating mutations $[9,10]$.

Therefore, given this background, MEK is a potential therapeutic target of interest for pharmacologic intervention in cancer. Inhibition of MEK has been shown to impair cell proliferation and impact a diverse array of cellular events including differentiation, apoptosis, and angiogenesis [11-15]. A number of MEK1/2 inhibitors are currently being investigated in the clinic across a range of cancers [16-19] including gynecologic malignancies [20], melanoma [17, 21], colorectal cancer [17], and acute myelogenous leukemia [22], with trametinib approved alone and in combination with the BRAF inhibitor dabrafenib for advanced metastatic melanoma with BRAF V600 mutations [23].

TAK-733 is an investigational, orally available, selective, non-ATP competitive, allosteric inhibitor of MEK $1 / 2$ with an $\mathrm{IC}_{50}$ for MEK signaling inhibition of 2-5 nM [24]. In the preclinical setting, TAK-733 has exhibited antitumor effects in vitro and in vivo against multiple cancer cell lines and xenograft models. For example, TAK-733 has demonstrated activity against multiple cutaneous melanoma cell lines, with a high proportion of $B R A F$ V600E-mutant cell lines showing high sensitivity $\left(\mathrm{IC}_{50}<0.1 \mu \mathrm{M}\right)$ and with no statistically significant association between BRAF status and response [25], and against uveal melanoma cell lines [26]. Additional studies have also shown tumor growth inhibition and regressions with TAK733 (dosed once daily) in human melanoma explant mouse models and mouse xenograft models [25, 27]. Synergistic activity was seen with TAK-733 in combination with the panRAF inhibitor TAK-632 in both $B R A F$-mutated melanoma cells and NRAS-mutated melanoma cells with acquired resistance to BRAF inhibitors [28]. Furthermore, antitumor activity has been reported in mesothelioma cell lines [29], and tumor growth suppression has also been seen in patient-derived colorectal cancer human tumor explants [30], and in mouse xenograft models of colorectal cancer [27] and lung cancer [31]. Pharmacokinetic data in mouse xenograft models indicate that plasma concentrations of TAK- 733 decrease rapidly $8-16 \mathrm{~h}$ after once-daily oral dosing [25]. Demonstrated pharmacodynamic effects of MEK inhibition with TAK-733 include decreases in phosphorylated ERK (pERK) [26], as seen in both sensitive and resistant melanoma cell lines and in tumor-bearing mice; elevated TAK-733 tumor concentrations were shown to correspond approximately with pERK reductions [25].
Based on these preclinical observations, this first-in-human, multicenter, open-label, phase I, dose-escalation study (NCT00948467) was conducted to investigate the safety, tolerability, pharmacokinetics, pharmacodynamics, and preliminary activity of TAK-733 in patients with solid tumors.

\section{Patients and methods}

\section{Patients}

Patients aged $\geq 18$ years with an Eastern Cooperative Oncology Group (ECOG) performance status of 0-2; a diagnosis of a nonhematologic malignancy for which there was no standard, curative, or life-prolonging treatment available; and with radiographically or clinically evaluable tumor (measurable disease as defined by the Response Evaluation Criteria for Solid Tumors [RECIST] was not a requirement during the dose-escalation stage) were eligible. Patients with ovarian or prostate cancer with elevated tumor markers (e.g. CA125 or prostate-specific antigen [PSA]) in the absence of measurable disease were also eligible. Patients also required adequate hematologic, renal, and hepatic function that were defined as: hemoglobin $\geq 9 \mathrm{~g} / \mathrm{dL}$; an absolute neutrophil count (ANC) $\geq 1500 / \mathrm{mm}^{3}$; a platelet count $\geq 100,000 / \mathrm{mm}^{3}$; a prothrombin time/international normalized ratio (INR) or activated partial thromboplastin time $\leq 1.5$ times the upper limit of normal (ULN); calculated creatinine clearance $\geq 50 \mathrm{~mL} / \mathrm{min}$; serum phosphorous or albumin-adjusted serum calcium $\leq \mathrm{ULN}$; bilirubin $\leq 1.5 \mathrm{x}$ ULN; and aspartate aminotransferase (AST), alanine aminotransferase (ALT), or alkaline phosphatase (ALP) $\leq 2.5 \times$ ULN (AST, ALT, and ALP may have been elevated up to $5 \mathrm{x}$ ULN if elevation could be reasonably ascribed to the presence of metastatic disease to liver and/or bone).

Patients who had major surgery within 14 days; strong or moderate CYP3A4 inhibitors/inducers within 14 days; antineoplastic therapy or radiotherapy within 21 days; any investigational product within 28 days; nitrosoureas or mitomycin C within 42 days; prior biologic or immunotherapy within 4 weeks; or prior ipilimumab within 4 months of first dose were not eligible. Patients were also excluded if they had symptomatic brain metastases; grade $\geq 2$ unresolved toxicity (except alopecia) from previous anticancer treatment; an ongoing or newly diagnosed eye abnormality putting the patient at risk for retinal vein thrombosis or central serous retinopathy; cardiovascular abnormalities including abnormal left ventricular ejection fraction, electrocardiogram, uncontrolled cardiovascular condition; or if they were receiving therapeutic anticoagulation.

Institutional review boards at each of the participating investigational centers approved the study, which was conducted in accordance with the ethical principles originating in or 
derived from the Declaration of Helsinki and its amendments and in accordance with 21 Code of Federal Regulations 50 / 56 / 312. All patients provided written informed consent.

\section{Study design}

This was an open-label, multicenter, first-in-human, phase I dose-escalation study. Patients were enrolled and treated at five sites in the United States from December 22, 2009, to April 30, 2013. The primary objectives were to evaluate the safety profile and determine the dose-limiting toxicities (DLTs), maximum tolerated dose (MTD), and recommended phase II dose (RP2D) of TAK-733, and to characterize the pharmacokinetics of TAK-733. The secondary objective was to evaluate antitumor activity. Exploratory objectives included investigating potential pharmacodynamic effects of TAK-733 in peripheral blood mononuclear cells, namely levels of pERK, and exploring pharmacokinetic/pharmacodynamic and pharmacokinetic/safety relationships.

Patients received oral TAK-733 once daily on days 1-21 of 28 -day treatment cycles. Dose escalation initially proceeded from a starting dose of $0.2 \mathrm{mg}$ in $100 \%$ increments using a single-patient cohort design (while allowing enrollment of additional eligible patients, up to four), until any patient experienced a DLT or a drug-related grade $\geq 3$ non-DLT adverse event (AE; except creatine kinase elevation) in cycle 1; or any patient experienced a TAK-733-related grade $\geq 2$ AE in cycle 1. Dose escalation then proceeded in $\leq 40 \%$ increments using a modified $3+3$ cohort design. No intra-patient dose escalation was permitted. The MTD was defined as the highest dose at which cycle 1 DLTs occurred in $0 / 3$ or $1 / 6$ patients. If fewer than 6 patients had been enrolled, the MTD dose level was expanded to a total of 6 patients.

DLTs were defined as any of the following considered possibly related to TAK-733: grade 4 neutropenia lasting $\geq 7$ consecutive days or grade $\geq 3$ neutropenia with fever/infection; grade 4 thrombocytopenia lasting $\geq 7$ consecutive days, platelet count $<10,000 / \mathrm{mm}^{3}$ at any time, or grade $\geq 3$ thrombocytopenia with clinically significant bleeding; grade 4 anemia; grade $\geq 3$ nausea/emesis despite optimal prophylaxis or grade $\geq 3$ diarrhea despite optimal antidiarrheal therapy; any other grade $\geq 3$ nonhematologic toxicity (except grade $\geq 3$ creatine kinase elevation considered not clinically significant); delay of $>2$ weeks in initiation of next cycle due to lack of adequate recovery of TAK-733-related toxicities; the inability to receive $\geq 75 \%$ of planned doses in a cycle due to TAK-733related toxicity; and any other grade $\geq 2$ TAK-733-related toxicities requiring dose reduction or discontinuation.

Following the initial dose-escalation stage, an expansion stage was planned at the MTD to investigate the safety, pharmacokinetics, pharmacodynamics, and antitumor activity of TAK-733 in patients with advanced unresectable melanoma. However, due to changes in the standard of care for melanoma treatment during the conduct of this study [32], coupled with a review of pharmacologic findings and the initial clinical findings on TAK-733 from the present study, a decision was made to cancel this stage of the study following thorough consideration by the sponsor.

\section{Assessments}

AEs were monitored throughout the trial and for 30 days after the last dose of study medication and were graded using the National Cancer Institute's Common Terminology Criteria for AEs version 3.0. Regular ophthalmologic examination was included in the safety monitoring. Response was assessed using the modified RECIST guideline (v1.1) [33]. Blood samples for determination of TAK-733 plasma concentrations were obtained at the following time points: pre-dose, and at $0.25,0.5,1,2,3,4,6,8,10$, and 24 h post-dose on days 1 and 21 of cycle 1; pre-dose on days 8 and 15 of cycle $1 ; 48,72,96$, and $120 \mathrm{~h}$ post-dose on day 21 of cycle 1; and pre-dose on day 1 of cycle 2 . Urine samples were collected over $0-24$ h postdose on day 21 of cycle 1 .

Plasma and urine samples were analyzed for TAK-733 concentrations using validated liquid chromatography-tandem mass spectrometry (LC-MS/MS) methods. The methods were applicable to the quantitation of TAK-733 within a dynamic range of $0.1-200 \mathrm{ng} / \mathrm{mL}$ for plasma samples and 5$10,000 \mathrm{ng} / \mathrm{mL}$ for urine samples. Quality controls, analyzed in duplicate over the validation range, and inter-assay precision, evaluated at each level in duplicate in six runs, met the performance and acceptance criteria. Noncompartmental analyses, using WinNonlin version 6.1 or higher (Pharsight, Cary, $\mathrm{NC}$ ), in pharmacokinetic-evaluable patients were used to determine plasma and urine pharmacokinetic parameters including: $\mathrm{C}_{\max }$, maximum plasma concentration; $\mathrm{T}_{\max }$, time to $\mathrm{C}_{\text {max }} ; \mathrm{AUC}_{0-\tau}$, area under the plasma concentration-time curve over the dosing interval; CL/F, apparent clearance; $t_{1 / 2}$, terminal elimination half-life; accumulation ratio; peak/trough ratio; and renal clearance.

Blood samples for pharmacodynamic analysis (pERK levels) were collected at the following time points: pre-dose and at $0.5,1,2,4,8$, and $24 \mathrm{~h}$ post-dose on days 1 and 21 of cycle 1 ; pre-dose on days 8 and 15 of cycle $1 ; 48,72,96$, and $120 \mathrm{~h}$ post-dose on day 21 of cycle 1 ; and pre-dose on day 1 of cycle 2. The extent of ERK phosphorylation in peripheral blood mononuclear cells was determined using an ex vivo stimulation assay; pERK levels in CD3-positive lymphocytes were measured by flow cytometry assessing mean fluorescence (MEFL) under phorbol myristate acetate (PMA)-stimulated and unstimulated conditions. Mean percent change from baseline in blood pERK level over time was determined following single and multiple doses of TAK-733. Noncompartmental analyses, using WinNonlin version 6.1 or higher (Pharsight, Cary, NC), in pharmacodynamic- 
evaluable patients were used to determine the following pharmacodynamic parameters for inhibition of ERK phosphorylation: maximum observed effect $\left(\mathrm{E}_{\max }\right)$, time to $\mathrm{E}_{\max }$ $\left(\mathrm{TE}_{\max }\right.$ ), area under the effect (inhibition)-time curve over the dosing interval $\left(\operatorname{AUEC}_{(0-\tau)}\right)$, and average effect over the dosing interval $\left(\mathrm{E}_{\mathrm{av}}\right)$.

\section{Statistics}

The safety population included all patients who received $\geq 1$ dose of TAK-733. The DLT-evaluable population included all patients who either experienced a DLT during cycle 1 or received $\geq 80 \%$ of scheduled cycle 1 doses without experiencing a DLT. The response-evaluable population included patients with measurable disease at baseline and $\geq 1$ postbaseline assessment. The pharmacokinetic-evaluable population included patients in the safety population for whom there were sufficient dosing and sufficient concentrationtime data to reliably estimate pharmacokinetic parameters. The pharmacodynamic-evaluable population included patients in the safety population who had sufficient dosing history and sufficient tumor and/or blood pharmacodynamic data available.

\section{Results}

\section{Patients}

Fifty one patients were enrolled and received $\geq 1$ dose of TAK-733. Patients' characteristics are summarized in Table 1 . The most common primary diagnoses included uveal melanoma (24\%), colon cancer (22\%), and cutaneous melanoma $(10 \%)$. Most $(90 \%)$ patients had received prior antineoplastic therapy, with $71 \%$ having received three or more prior therapies. Although not an exclusion criterion for the dose-escalation portion of the study, no patients were reported to have been previously treated with inhibitors of the MAPK pathway. Thirty nine (76\%) patients discontinued treatment due to progressive disease, $7(14 \%)$ due to AEs, 2 (4\%) each due to symptomatic deterioration and patient withdrawal, and 1 (2\%) for non-compliance.

\section{Dose escalation, DLTs, and MTD determination}

Fifty one patients received TAK-733 at one of eleven dose levels: $0.2 \mathrm{mg}(n=1) ; 0.4 \mathrm{mg}(n=1) ; 0.8 \mathrm{mg}(n=2)$; $1.6 \mathrm{mg}(n=2) ; 3.2 \mathrm{mg}(n=4) ; 4.4 \mathrm{mg}(n=4) ; 6 \mathrm{mg}$ $(n=4) ; 8.4 \mathrm{mg}(n=9) ; 11.8 \mathrm{mg}(n=8) ; 16 \mathrm{mg}(n=9)$; and $22 \mathrm{mg}(n=7)$. Forty one patients were included in the DLTevaluable population. No DLTs were observed in patients who received TAK-733 in the first eight dose cohorts $(0.2-8.4 \mathrm{mg})$.
Table 1 Baseline patient demographics and disease characteristics. ECOG, Eastern Cooperative Oncology Group; NSCLC, non-small cell lung cancer

\begin{tabular}{ll}
\hline Characteristic & $N=51$ \\
\hline Median age, years (range) & $58(24-75)$ \\
Male, $n(\%)$ & $26(51)$ \\
Race, $n(\%)$ & \\
White & $42(82)$ \\
Black or African American & $8(16)$ \\
Not reported & $1(2)$ \\
ECOG performance status, $n(\%)$ & \\
0 & $22(43)$ \\
1 & $29(57)$ \\
Disease primary diagnosis, $n(\%)$ & \\
Melanoma uveal & $12(24)$ \\
Colon cancer & $11(22)$ \\
Melanoma of the skin & $5(10)$ \\
Other melanoma* & $4(8)$ \\
NSCLC & $3(6)$ \\
Anal cancer & $2(4)$ \\
Colorectal cancer & $2(4)$ \\
Rectal cancer & $2(4)$ \\
Other & \\
Prior therapy, $n$ (\%) & $10(20)$ \\
Prior surgery or non-radiation procedure & \\
Prior radiation & \\
Prior antineoplastic therapy & \\
1 & $50(98)$ \\
2 & $38(75)$ \\
Best response to last prior antineoplastic therapy, $n(\%)$ & \\
Partial response & $46(90)$ \\
Stable disease & $7(14)$ \\
Unknogressive disease & $3(6)$ \\
\hline
\end{tabular}

*Melanoma of the scapular, ocular malignant melanoma, ocular melanoma, and melanoma: unknown, each $\mathrm{n}=1$. †Adrenal, bladder, head and neck, liver, ovarian, and skin cancer, melanoma, sarcoma, unknown highgrade malignant neoplasm, and unknown primary cancer, each $n=1$

Subsequently, 4 patients experienced DLTs in cycle 1. In the $11.8 \mathrm{mg}$ cohort, one of six DLT-evaluable patients had a DLT of grade 3 dermatitis acneiform on day 21 of cycle 1 . The patient received oral minocycline, topical Neosporin, and topical clindamycin, and TAK-733 was discontinued; the event resolved approximately 1 week later. In the $16 \mathrm{mg}$ cohort, one of seven DLT-evaluable patients had a DLT of grade 3 dermatitis acneiform on day 21 . The patient received oral minocycline, topical clindamycin, and topical hydrocortisone, and TAK-733 was discontinued; the event was reported as ongoing at last follow-up. In the $22 \mathrm{mg}$ cohort, one patient 
had grade 3 fatigue, pustular rash, and dermatitis acneiform on day 21 of cycle 1; TAK-733 was discontinued and the events resolved in approximately 2 weeks. A second patient had grade 2 dermatitis acneiform and stomatitis on day 12 of cycle 1; the TAK-733 dose was reduced to $16 \mathrm{mg}$ and the events resolved in 3 and 8 weeks, respectively.

Based on the observed DLTs in cycle 1, the MTD of TAK733 was determined to be $16 \mathrm{mg}$ once daily on days $1-21$ in 28-day treatment cycles.

\section{TAK-733 exposure and safety profile}

Patients received a median of 2 cycles (range 1-11) of TAK$733 ; 7(14 \%)$ patients received $\geq 6$ cycles. The mean total dose of TAK-733 received was $581.66 \mathrm{mg}$ (ranging from $4.2 \mathrm{mg}$ in the $0.2 \mathrm{mg}$ dose level to $1425.71 \mathrm{mg}$ in the $22.0 \mathrm{mg}$ dose level), with a mean dose per week of $57.71 \mathrm{mg}$ (ranging from 1.34 to $112.64 \mathrm{mg}$ ).

All patients experienced at least one AE of any grade, and $88 \%$ reported drug-related AEs (Table 2). Grade $\geq 3$ AEs were reported in $27(53 \%)$ patients, with drug-related grade $\geq 3$ AEs reported in $12(24 \%)$. AEs of any grade reported in at least $20 \%$ of patients, drug-related AEs reported in at least $10 \%$ of patients, and all drug-related grade $\geq 3 \mathrm{AEs}$, are listed in Table 2.

Overall, $34(67 \%)$ patients experienced any rash AEs. This included $28(55 \%)$ with dermatitis acneiform, $3(6 \%)$ with rash, 2 (4\%) each with macular rash, maculo-papular rash, and papular rash, and $1(2 \%)$ each with exfoliative rash, erythematous rash, pruritic rash, and pustular rash. Two of the rash DLTs are shown in Fig. 1. The number of patients who experienced rash AEs increased with increasing dose of TAK-733; however, an analysis was conducted indicating no relationship between TAK-733 exposure and skin AEs (data not shown). No cutaneous squamous cell carcinomas were seen.

A total of 7 (14\%) patients experienced ophthalmic AEs within the eye disorders system organ class, including 1 at $8.4 \mathrm{mg}, 2$ at $16 \mathrm{mg}$, and 4 at $22 \mathrm{mg}$. These included visual impairment $(n=3)$, photopsia $(n=2)$, blurred vision $(n=2)$, abnormal sensation in eye, photophobia, periorbital edema, and retinal edema (each $n=1$ ).

A total of $14(27 \%)$ patients experienced at least one serious AE (SAE), including 1 ( $2 \%)$ who experienced drugrelated SAEs. The only SAEs reported in more than one patient were progression of metastatic melanoma $(n=3)$, pulmonary embolism $(n=2)$, and anemia $(n=2)$. One patient experienced SAEs of hydroureter, hydronephrosis, and pulmonary embolism that were assessed as possibly related to TAK-733; the patient had a history of hydroureter and hydronephrosis at study entry.

Seven $(14 \%)$ patients experienced AEs that required TAK733 discontinuation; these included: stomatitis in 1 patient at
Table 2 Safety profile of TAK-733, including AEs of any grade reported in at least $20 \%$ of patients, drug-related AEs reported in at least $10 \%$ of patients, and all drug-related grade $\geq 3$ AEs. AE, adverse event; AST, aspartate aminotransferase; CPK, creatine phosphokinase

\begin{tabular}{|c|c|}
\hline $\mathrm{AE}, n(\%)$ & $N=51$ \\
\hline Any AE & $51(100)$ \\
\hline \multicolumn{2}{|l|}{ Common AEs (any grade; $\geq 20 \%$ of patients) } \\
\hline Dermatitis acneiform & $28(55)$ \\
\hline Diarrhea & $19(37)$ \\
\hline Fatigue & $18(35)$ \\
\hline Peripheral edema & $14(27)$ \\
\hline Increased AST & $13(25)$ \\
\hline Increased CPK & $10(20)$ \\
\hline Decreased appetite & $10(20)$ \\
\hline Any drug-related $\mathrm{AE}$ & $45(88)$ \\
\hline \multicolumn{2}{|l|}{ Common drug-related $\mathrm{AE}(\geq 10 \%$ of patients $)$} \\
\hline Dermatitis acneiform & $26(51)$ \\
\hline Diarrhea & $15(29)$ \\
\hline Increased blood CPK & $10(20)$ \\
\hline Fatigue & $9(18)$ \\
\hline Stomatitis & $9(18)$ \\
\hline Peripheral edema & $8(16)$ \\
\hline AST increased & $7(14)$ \\
\hline Dry skin & $5(10)$ \\
\hline Any grade $\geq 3 \mathrm{AE}$ & $27(53)$ \\
\hline Any drug-related grade $\geq 3 \mathrm{AE}$ & $12(24)$ \\
\hline \multicolumn{2}{|l|}{ Drug-related grade $\geq 3$ AEs } \\
\hline Increased blood CPK & $5(10)$ \\
\hline Dermatitis acneiform & $4(8)$ \\
\hline Stomatitis & $1(2)$ \\
\hline Fatigue & $1(2)$ \\
\hline Rash pustular & $1(2)$ \\
\hline Hypophosphatemia & $1(2)$ \\
\hline Pain in extremity & $1(2)$ \\
\hline Neuropathy peripheral & $1(2)$ \\
\hline Pulmonary embolism & $1(2)$ \\
\hline Any serious $\mathrm{AE}$ & $14(27)$ \\
\hline Any drug-related serious $\mathrm{AE}$ & $1(2)$ \\
\hline AE resulting in study drug discontinuation & $7(14)$ \\
\hline On-study death & $5(10)$ \\
\hline
\end{tabular}

$8.4 \mathrm{mg}$; dermatitis acneiform in 1 patient at $11.8 \mathrm{mg}$ and 1 patient at $16 \mathrm{mg}$; pneumonia in 1 patient at $16 \mathrm{mg}$; seborrheic dermatitis, swelling face, and fatigue in 1 patient at $16 \mathrm{mg}$; fatigue, pustular rash, and dermatitis acneiform (DLT) in 1 patient at $22 \mathrm{mg}$; and bacteremia in 1 patient at $22 \mathrm{mg}$. All AEs were considered related to TAK-733 except for pneumonia and bacteremia.

Five patients died on-study. None of the deaths were considered related to TAK-733, and all were due to the progression of the patients' respective malignancies. 


\section{Pharmacokinetics}

TAK-733 plasma concentration-time data were available from all 51 patients who received TAK-733 over the dose range $0.2-22 \mathrm{mg}$; plasma concentration-time profiles on day 1 and day 21 of cycle 1 are shown in Fig. 2a and b. Following oral administration, absorption was fast, with an overall median $\mathrm{T}_{\max }$ of $3 \mathrm{~h}$ (range $0.5-8.1$ ) (Fig. 2a, b, Supplementary Table S1). Terminal half-life following multiple dosing ranged from 29 to $50 \mathrm{~h}$ over the $11.8-22 \mathrm{mg}$ dose range, peak/trough ratio ranged from 1.3 to $4.8(0.2-22 \mathrm{mg}$ dose range), and the accumulation ratio varied from 0.9 to 9.0 across the $0.2-$ $22 \mathrm{mg}$ dose range. Dose-proportionality analysis of TAK733 steady-state systemic exposure $\left(\mathrm{AUC}_{0-\tau}\right)$ versus dose showed a point estimate of the slope of 0.8 (95\% CI: 0.63 , 0.98 ), indicating less than a dose-proportional increase over the TAK-733 dose range of 0.2-22 $\mathrm{mg}$ (Fig. 2c).

Urine pharmacokinetic data were available from 16 patients for estimation of the renal clearance of TAK-733. Renal clearance ranged from 0.05 to $0.49 \mathrm{~L} / \mathrm{h}$, which was $0.5 \%$ to $4.8 \%$ of the apparent oral clearance over the $0.2-$ $22 \mathrm{mg}$ dose range.

\section{Pharmacodynamics}

Data on the pharmacodynamic effects of TAK-733 (inhibition of ERK phosphorylation in peripheral blood mononuclear cells, as a biomarker of MEK inhibition) were available for 46 patients. Mean percent changes from baseline in blood
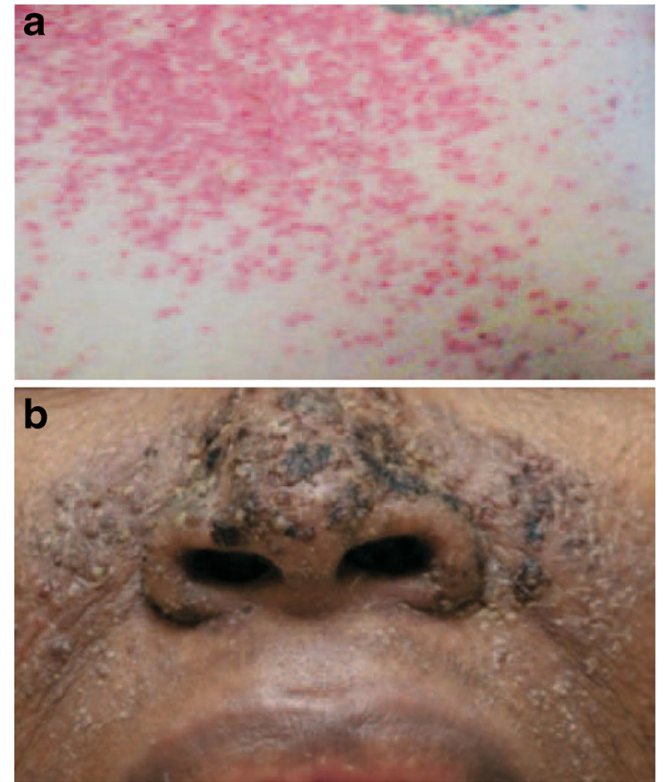

Fig. 1 Dose-limiting toxicities (DLTs) of rash seen in patients receiving TAK-733. a Grade 3 dermatitis acneiform on day 21 of cycle 1 in a patient receiving TAK-733 $11.8 \mathrm{mg}$ - painful rash seen on scalp, face, and chest (shown in photo). b Grade 2 rash in a patient receiving TAK$73322 \mathrm{mg}$ that required dose reduction in cycle 1
pERK levels following single and multiple TAK-733 doses are shown in Fig. 3a, b. $\mathrm{TE}_{\max }$ ranged from 0.8 to $24 \mathrm{~h}$, with maximum decrease in pERK at day 21 of $46-97 \%\left(E_{\max }\right)$ in patients receiving doses of $8.4 \mathrm{mg}$ and above. On day 21 of cycle 1 , the duration of $\mathrm{pERK}$ decreases appeared relatively transient at TAK-733 doses of less than $8.4 \mathrm{mg}$, while at higher doses target inhibition appeared to be sustained beyond $4 \mathrm{~h}$ (Fig. 3a, b). At the MTD of $16 \mathrm{mg}$, mean $\mathrm{E}_{\max }$ was $85 \%$, and mean $\operatorname{AUEC}_{(0-\tau)}$ was $1947 \mathrm{~h} * \%$ on day 21 of cycle 1, translating to a mean $\mathrm{E}_{\mathrm{av}}$ of $82 \%$ (Table 3 ). Individual and mean values of $E_{\max }$ and time-averaged effect by TAK-733 dose at steady state (day 21) are shown in Fig. 3c, d, respectively. No analyses of correlations between pERK decreases and antitumor effects and no pharmacodynamic analyses at tumor sites were conducted as part of this study.

\section{Antitumor activity}

Among 41 response-evaluable patients, 2 (5\%) patients had partial responses. One patient (male, age 68 years) with BRAF L597R cutaneous melanoma, who received TAK-733 at $16 \mathrm{mg}$, had a partial response that was reported at cycle 4 and maintained until cycle 8 (approximate duration of 4 months). The patient had received three prior lines of therapy with interferon, granulocyte macrophage colonystimulating factor (both as adjuvant therapy), and dacarbazine, as well as prior radiation therapy. A second patient (male, age 68 years) with cutaneous melanoma (mutation status not feasible to determine), who received TAK-733 at $22 \mathrm{mg}$, had a partial response at cycle 2 that was maintained until cycle 6 (approximate duration of 4 months). The patient had received one prior line of therapy with ipilimumab, to which his best response was progressive disease.

A further $15(37 \%)$ patients had a best response of stable disease, and the other 24 (59\%) evaluable patients had progressive disease. Two patients with melanoma who received TAK-733 at the $8.4 \mathrm{mg}$ and $22 \mathrm{mg}$ dose levels experienced stable disease lasting for 6 months or longer.

With a very small sample size, and low rate of responders, no correlation of mutation profile to response was seen in an analysis of archival tumor biopsies (data not shown).

\section{Discussion}

This was the first-in-human study of TAK-733 in patients with advanced solid tumors. Our findings showed that the safety profile of TAK-733 was generally acceptable, with a manageable toxicity profile up to a dose of $16 \mathrm{mg}$ QD for 21 days in 28-day cycles, which was determined to be the MTD $(n=9)$. Results from pharmacokinetic analyses showed that less than dose-proportional increases in steady-state exposures of TAK733 were observed over the dose range of $0.2-22 \mathrm{mg}$, and, 
Fig. 2 Pharmacokinetics of TAK-733 in the pharmacokineticevaluable population. Mean TAK-733 plasma concentrationtime profiles on $\mathbf{a}$ day 1 and $\mathbf{b}$ day 21 of cycle 1 , and $\mathbf{c}$ TAK-733 steady-state systemic exposure (day $21 \mathrm{AUC}_{0-\tau}$ ) versus dose (slope 0.8, $95 \%$ CI: 0.63-0.98) indicating a less than dose-proportional relationship

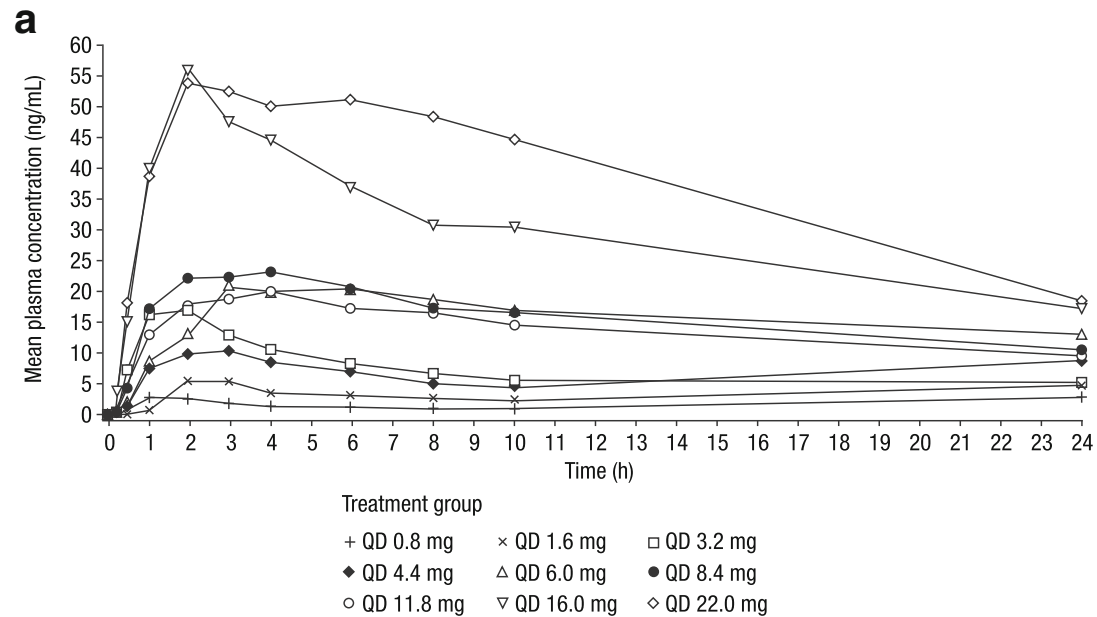

b
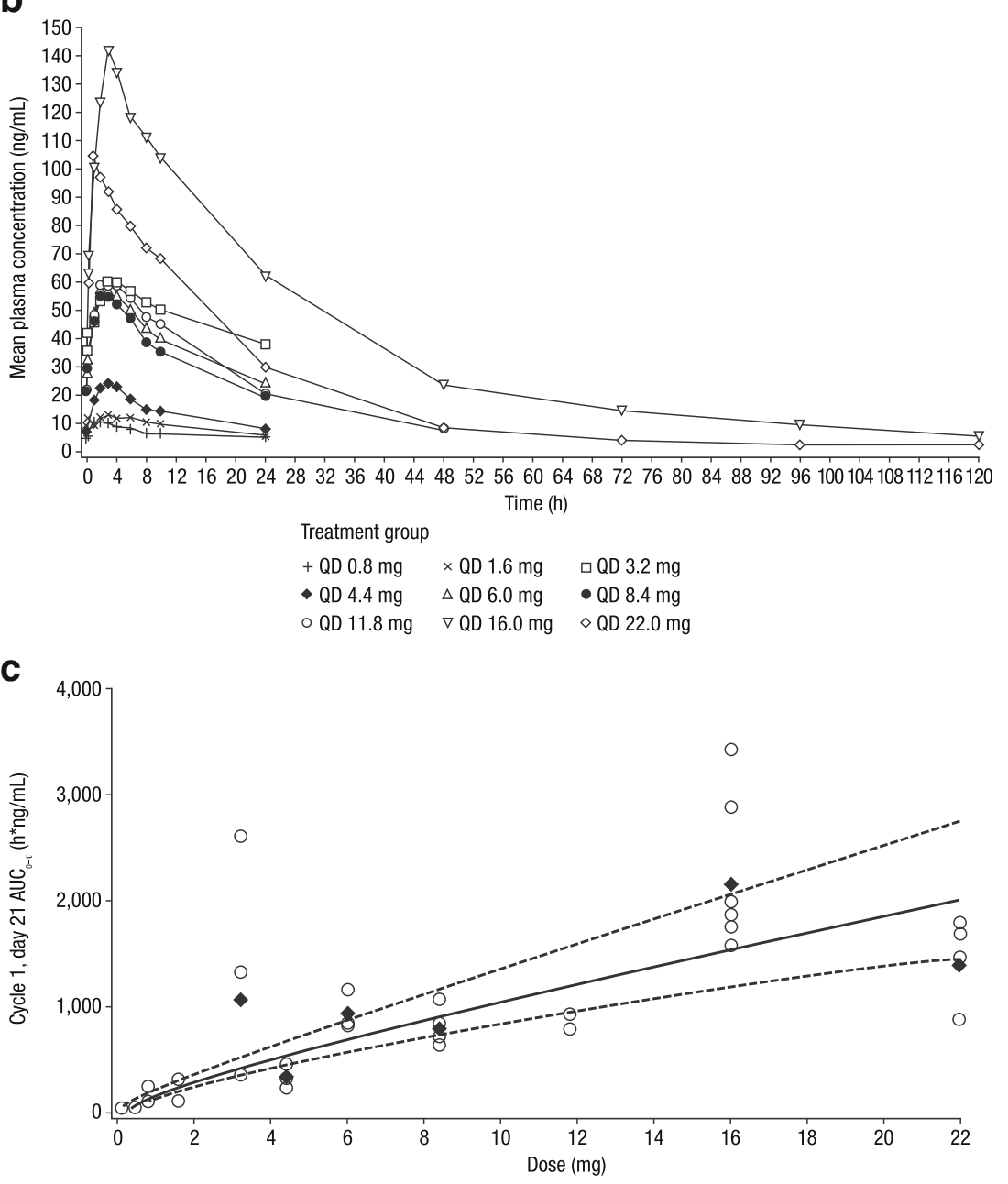

- Observed $(n=32)$

- Geometric mean

- Predicted

---- 95\% confidence interval therefore, variability in dose-normalized exposures could not be assessed. A key finding of importance from the study was that sustained decreases in blood pERK levels were seen at higher doses of TAK-733; this anticipated pharmacodynamic effect of TAK-733 supports the mechanism of action of MEK1/2 inhibition. Also of note, we showed that two patients 

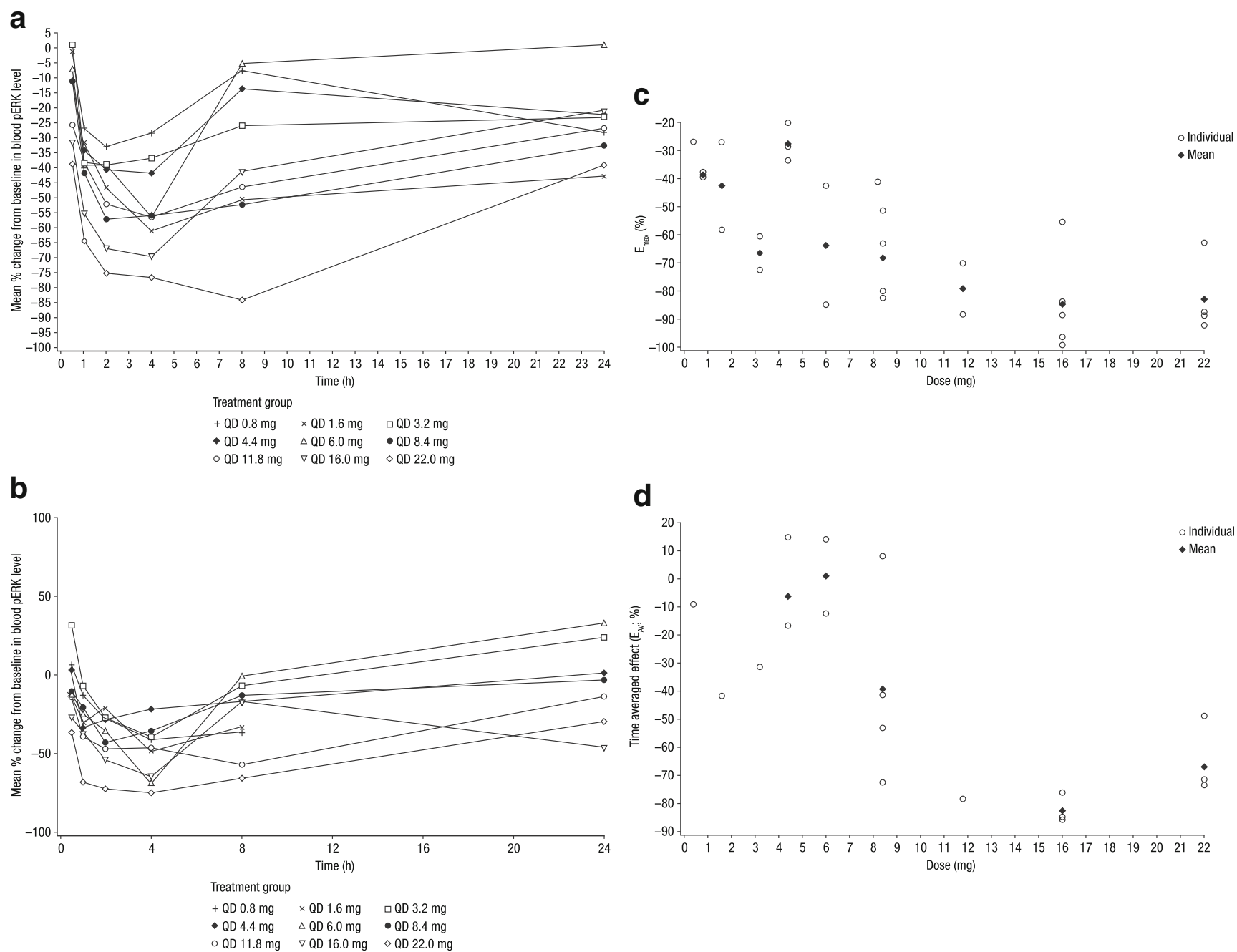

Fig. 3 Pharmacodynamics of TAK-733 in the pharmacodynamicevaluable population. Mean percent change from baseline in blood $\mathrm{pERK}$ levels versus time profiles ( $0-24 \mathrm{~h})$ on a day 1 and $\mathbf{b}$ day 21 of cycle 1 , and

with cutaneous melanoma, including one with a BRAF L597R mutation, achieved partial responses that lasted approximately 4 months, suggesting evidence of preliminary antitumor activity with TAK-733.

TAK-733 represents one of several MEK $1 / 2$ inhibitors being investigated for the treatment of various cancers [16, 34]. Like the approved agent trametinib, as well as selumetinib [35] and other investigational MEK1/2 inhibitors, TAK-733 is a non-ATP competitive allosteric inhibitor of the MEK1 and MEK2 BRAF substrates [24]. The findings from the present phase I study appear generally consistent with data from early-phase clinical trials of these other MEK $1 / 2$ inhibitors, which, with the exception of trametinib, have generally demonstrated limited single-agent activity across multiple tumor types, along with similar DLTs and other toxicities [16, 34]. For example, the DLTs and/or most common drug-related AEs with TAK-733 in the present phase I study included dermatitis acneiform, diarrhea, increased blood creatine phosphokinase (CPK), fatigue, and stomatitis. These are similar to the

relationship between dose and (c) maximal pERK decrease $\left(\mathrm{E}_{\max }\right)$ and (d) steady-state time-averaged effect $\left(\mathrm{E}_{\mathrm{av}}\right)$ on day 21 of cycle 1

common toxicities reported with other MEK1/2 inhibitors including trametinib [36-38], selumetinib [19], RO4987655 [39], and PD-0325901 [40]; trametinib has been reported to be specifically associated with acneiform eruptions [41]. Similarly, DLTs in other phase I studies of MEK1/2 inhibitors have included rash, diarrhea, increased blood CPK, and ocular toxicities with ARRY-424704, refametinib, cobimetinib, ARRY-438162, and pimasertib [16, 34]. These findings reflect the general association of dermatologic toxicities with smallmolecule targeted cancer therapies such as kinase inhibitors, including MEK inhibitors [42, 43].

A notable aspect of the safety profile of TAK-733 was the limited rate of ophthalmic AEs, and the absence of retinopathies in the present study. Central serous-like retinopathy and serous retinal detachment, retinal vein occlusion, blurred vision, transient visual disturbance, and retinal pigment epithelial detachment have all been reported as DLTs with other MEK1/2 inhibitors in phase I clinical trials [16], with transient drug-related retinopathies reported with MEK inhibitors in 
Table 3 Key TAK-733 pharmacodynamic parameters of decreases in blood pERK levels following oral administration of TAK-733 at doses of 0.8 to $22 \mathrm{mg}$ in the pharmacodynamic-evaluable population. $\mathrm{AUEC}_{(0-\tau)}$, area under the effect (inhibition of ERK phosphorylation)-time curve over

the dosing interval; $\mathrm{E}_{\mathrm{av}}$, average effect (inhibition of ERK phosphorylation) over the dosing interval; $\mathrm{E}_{\max }$, maximum observed effect of inhibition of ERK phosphorylation; NR, not reported as $\mathrm{n}<2$; SD, standard deviation; $\mathrm{TE}_{\max }$, time to $\mathrm{E}_{\max }$

\begin{tabular}{|c|c|c|c|c|c|c|}
\hline Dose, mg & $N$ & Day & $\begin{array}{l}E_{\max }, \% \\
\text { Mean (SD) }\end{array}$ & $\begin{array}{l}\mathrm{TE}_{\max }, \mathrm{h} \\
\text { Median (range) }\end{array}$ & $\begin{array}{l}\operatorname{AUEC}_{(0-\tau)}, \mathrm{h}^{* \%} \\
\text { Mean (SD) }\end{array}$ & $\begin{array}{l}\mathrm{E}_{\mathrm{av}}, \% \\
\text { Mean (SD) }\end{array}$ \\
\hline \multirow[t]{2}{*}{0.8} & \multirow[t]{2}{*}{2} & 1 & $-40.55(2.5)$ & $3.0(2-4)$ & $-450(60)$ & $-19.3(2.0)$ \\
\hline & & 21 & $-39.2(1.1)$ & $3.0(2-4)$ & NR & NR \\
\hline \multirow[t]{2}{*}{1.6} & \multirow[t]{2}{*}{2} & 1 & $-61.3(7.0)$ & $4.0(4-4)$ & $-1175(134)$ & $-47(8.2)$ \\
\hline & & 21 & $-43.0(21.9)$ & NR & -1020 & -42.2 \\
\hline \multirow[t]{2}{*}{3.2} & \multirow[t]{2}{*}{3} & 1 & $-41.2(7.2)$ & $1.1(1-4)$ & $-643(405)$ & $-26.9(16.9)$ \\
\hline & & 21 & $-66.8(8.4)$ & NR & -785 & -31.6 \\
\hline \multirow[t]{2}{*}{4.4} & \multirow[t]{2}{*}{4} & 1 & $-49.2(3.7)$ & $1.5(1-2)$ & $-542(492)$ & $-22.5(20.5)$ \\
\hline & & 21 & $-28.0(6.8)$ & $2.0(2-4)$ & $-164(447)$ & $-6.4(18.3)$ \\
\hline \multirow[t]{2}{*}{6} & \multirow[t]{2}{*}{3} & 1 & $-59.8(32.7)$ & $4.0(1-4)$ & $-397(458)$ & $-16.5(18.9)$ \\
\hline & & 21 & $-64.0(29.6)$ & NR & $29(464)$ & $0.7(18.7)$ \\
\hline \multirow[t]{2}{*}{8.4} & \multirow[t]{2}{*}{8} & 1 & $-64.5(14.5)$ & $3.0(1-8)$ & $-981(624)$ & $-41.6(26.5)$ \\
\hline & & 21 & $-63.8(17.6)$ & $2.1(1-4)$ & $-937(811)$ & $-39.7(34.2)$ \\
\hline \multirow[t]{2}{*}{11.8} & \multirow[t]{2}{*}{7} & 1 & $-61.3(11.4)$ & $4.1(4-8)$ & $-971(241)$ & $-40.5(10.0)$ \\
\hline & & 21 & $-79.3(12.7)$ & $2.2(2.1-2.3)$ & -1900 & -78.1 \\
\hline \multirow[t]{2}{*}{16} & \multirow[t]{2}{*}{9} & 1 & $-77.3(6.7)$ & $4.0(2-8)$ & $-948(624)$ & $-40.1(26.3)$ \\
\hline & & 21 & $-84.7(17.3)$ & $4.6(4-24)$ & $-1947(125)$ & $-82.4(5.5)$ \\
\hline \multirow[t]{2}{*}{22} & \multirow[t]{2}{*}{7} & 1 & $-84.1(9.0)$ & $7.6(1-8)$ & $-1517(257)$ & $-64.5(11.3)$ \\
\hline & & 21 & $-82.9(13.4)$ & $3.1(1-8)$ & $-1615(288)$ & $-66.7(11.7)$ \\
\hline
\end{tabular}

For $\operatorname{AUEC}_{(0-\tau)}, \mathrm{E}_{\mathrm{av}}$, and $\mathrm{E}_{\mathrm{max}}$, the parameters are based on \% change from baseline in pERK levels versus time, with inhibition of ERK phosphorylation being greater the more negative the number

patients with metastatic melanoma $[37,44]$ and MEK inhibitor treatment associated with other ocular adverse events [45]. These ocular toxic effects appear unique to MEK1/2 inhibitors [16], but did not appear to form a notable component of the TAK-733 safety profile.

For determination of plasma and urine pharmacokinetic profile, a validated LC-MS/MS method was employed and the TAK-733 concentrations were within the dynamic ranges of the assay $(0.1-200 \mathrm{ng} / \mathrm{mL}$ for plasma and 5-10,000 $\mathrm{ng} / \mathrm{mL}$ for urine). Findings from the analyses of TAK-733 pharmacokinetics in the present study indicated that exposures were attained that were in excess of those associated with antitumor activity in preclinical studies. For example, at the $16 \mathrm{mg}$ dose of TAK-733, the geometric mean steady-state exposure (day $\left.21 \mathrm{AUC}_{0-\tau}\right)$ of $2154.4 \mathrm{~h} * \mathrm{ng} / \mathrm{mL}$ was approximately two-fold higher than the exposures associated with stasis in the most sensitive xenograft model treated with single-agent TAK-733 (861-1065 h*ng/mL) [46]. Furthermore, pharmacodynamic findings also showed that the mean $\mathrm{E}_{\mathrm{av}}$ for inhibition of blood ERK phosphorylation at $16 \mathrm{mg}(82 \%)$ exceeded the lower bound of time-averaged inhibition of ERK phosphorylation (76-89\%) associated with tumor stasis in xenograft models treated with single-agent TAK-733 [46]. As was planned in the cancelled expansion stage, a more informative measure of the pharmacodynamic effects of TAK-733 would have been to measure its impact on pERK and downstream outcome markers (e.g. markers of proliferation and apoptosis) in matched tumor biopsies. Similar pharmacodynamic effects of target pathway inhibition, i.e. a decrease in pERK levels in peripheral blood mononuclear cells, malignant cells, and/or paired tumor biopsies, have been reported with other MEK1/2 inhibitors, including trametinib [37], selumetinib (79\% geometric mean pERK reduction in paired tumor biopsies [19, 22]), ARRY-424704 [47], and RO4987655 [39], and with the dual Raf/MEK inhibitor RO5126766 [48], although no correlations with response have been reported.

As with the safety profile and pharmacodynamic effects, the antitumor activity reported with TAK-733 in the present study appears consistent with that seen with most other MEK1/2 inhibitors in early-phase development [16, 34]. The objective response rate was limited, at $5 \%$, with responses including two partial responses in patients with cutaneous melanoma; additionally, two patients with melanoma achieved stable disease lasting for 6 months or longer. Notably, the response in a patient with BRAF L597R mutant melanoma is consistent with observations with other MEK1/2 inhibitors, including trametinib [36-38], which have shown activity in BRAF-mutant melanoma. Specifically, trametinib has been approved by the US FDA for the treatment of BRAF V600E/K-mutant melanoma, having demonstrated substantial 
response rates of approximately $20 \%[36,38]$ and improved progression-free survival and overall survival compared to chemotherapy with dacarbazine or paclitaxel [36] in this patient population. Subsequently, the combination of MEK1/2 inhibition with trametinib and the BRAF inhibitor dabrafenib has been FDA-approved based on enhanced responses and progression-free survival compared to dabrafenib monotherapy and improved overall survival compared to vemurafenib monotherapy $[49,50]$, indicating the importance of dual inhibition of the Ras/Raf/MEK/ERK pathway for improving antitumor activity, ameliorating paradoxical activation of MAPK signaling and partially overcoming potential resistance to single-target inhibition, e.g. BRAF inhibition.

In conclusion, the findings of the present phase I study of TAK-733 have demonstrated a safety profile, pharmacodynamic effects, and antitumor activity consistent with other MEK1/2 inhibitors, with key toxicities including dermatitis acneiform, diarrhea, increased blood CPK, fatigue, and stomatitis, and limited single-agent antitumor activity, including a partial response in a patient with BRAF-mutant melanoma. Given the limited antitumor activity, and in the context of data with trametinib and the recent changes in the standards of care in this indication, there are no plans for the future development of TAK-733 in patients with melanoma, and there are currently no other ongoing clinical studies of TAK-733.

Acknowledgments The authors would like to thank all the patients who participated in this study and their families, as well as staff at all investigational sites. The authors would also like to thank Steve Hill of FireKite, an Ashfield company, part of UDG Healthcare plc, for writing support during the development of this manuscript, which was funded by Millennium Pharmaceuticals, Inc. Employees of the sponsor, Millennium Pharmaceuticals, Inc., a wholly owned subsidiary of Takeda Pharmaceutical Company Limited, participated in trial design, data collection, data analysis, data interpretation, and writing of the report. The sponsor of the study was involved in the design of the trial and provided grants to trial sites and had no other involvement in conduct of the trial. The corresponding author had full access to all the data in the study and had final responsibility for the decision to submit for publication.

\section{Compliance with ethical standards}

Conflicts of interest AR has acted as a consultant for and has received honoraria from Amgen, Compugen, Flexus, GSK, Genentech, and Merck, and holds stock in Novartis and Kite Pharma. JAS has acted as a consultant for Millennium Pharmaceuticals, Inc., Merck, and Amgen, and has received honorarium from Millennium Pharmaceuticals, Inc. The institution of JAS has also received grants from Bristol-Myers Squibb, GSK, and Millennium Pharmaceuticals, Inc. XZ, SF, RN, MK, EG and VB were employees of Millennium Pharmaceuticals, Inc., a wholly owned subsidiary of Takeda Pharmaceutical Company Limited. AAA, PL, AP, and GKD have no conflicts of interest to disclose.

Funding The work was supported by Millennium Pharmaceuticals, Inc., Cambridge, MA, USA, a wholly owned subsidiary of Takeda Pharmaceutical Company Limited.
Ethical approval All procedures performed in studies involving human participants were in accordance with the ethical standards of the institutional and/or national research committee and with the 1964 Helsinki declaration and its later amendments or comparable ethical standards.

Informed consent Informed consent was obtained from all individual participants included in the study.

Open Access This article is distributed under the terms of the Creative Commons Attribution 4.0 International License (http:// creativecommons.org/licenses/by/4.0/), which permits unrestricted use, distribution, and reproduction in any medium, provided you give appropriate credit to the original author(s) and the source, provide a link to the Creative Commons license, and indicate if changes were made.

\section{References}

1. Frémin C, Meloche S (2010) From basic research to clinical development of MEK1/2 inhibitors for cancer therapy. J Hematol Oncol 3:8

2. Santarpia L, Lippman SM, El-Naggar AK (2012) Targeting the MAPK-RAS-RAF signaling pathway in cancer therapy. Expert Opin Ther Targets 16(1):103-119

3. Roberts PJ, Der CJ (2007) Targeting the Raf-MEK-ERK mitogenactivated protein kinase cascade for the treatment of cancer. Oncogene 26(22):3291-3310

4. Sebolt-Leopold JS, Herrera R (2004) Targeting the mitogenactivated protein kinase cascade to treat cancer. Nat Rev Cancer 4(12):937-947

5. Bucheit AD, Syklawer E, Jakob JA, Bassett RL Jr, Curry JL, Gershenwald JE, Kim KB, Hwu P, Lazar AJ, Davies MA (2013) Clinical characteristics and outcomes with specific BRAF and NRAS mutations in patients with metastatic melanoma. Cancer 119(21):3821-3829

6. Fernandez-Medarde A, Santos E (2011) Ras in cancer and developmental diseases. Genes Cancer 2(3):344-358

7. Fearon ER, Vogelstein B (1990) A genetic model for colorectal tumorigenesis. Cell 61(5):759-767

8. Vogelstein B, Fearon ER, Hamilton SR, Kern SE, Preisinger AC, Leppert M, Nakamura Y, White R, Smits AM, Bos JL (1988) Genetic alterations during colorectal-tumor development. N Engl J Med 319(9):525-532

9. Pratilas CA, Solit DB (2007) Therapeutic strategies for targeting BRAF in human cancer. Rev Recent Clin Trials 2(2):121-134

10. Sclafani F, Gullo G, Sheahan K, Crown J (2013) BRAF mutations in melanoma and colorectal cancer: a single oncogenic mutation with different tumour phenotypes and clinical implications. Crit Rev Oncol Hematol 87(1):55-68

11. Collisson EA, De A, Suzuki H, Gambhir SS, Kolodney MS (2003) Treatment of metastatic melanoma with an orally available inhibitor of the Ras-Raf-MAPK cascade. Cancer Res 63(18):5669-5673

12. Cragg MS, Jansen ES, Cook M, Harris C, Strasser A, Scott CL (2008) Treatment of B-RAF mutant human tumor cells with a MEK inhibitor requires Bim and is enhanced by a $\mathrm{BH} 3$ mimetic. J Clin Invest 118(11):3651-3659

13. Hoshino R, Tanimura S, Watanabe K, Kataoka T, Kohno M (2001) Blockade of the extracellular signal-regulated kinase pathway induces marked G1 cell cycle arrest and apoptosis in tumor cells in which the pathway is constitutively activated: up-regulation of p27(Kip1). J Biol Chem 276(4):2686-2692 
14. McCubrey JA, Milella M, Tafuri A, Martelli AM, Lunghi P, Bonati A, Cervello M, Lee JT, Steelman LS (2008) Targeting the Raf/ MEK/ERK pathway with small-molecule inhibitors. Curr Opin Investig Drugs 9(6):614-630

15. Vella LJ, Pasam A, Dimopoulos N, Andrews M, Knights A, Puaux AL, Louahed J, Chen W, Woods K, Cebon JS (2014) MEK inhibition, alone or in combination with BRAF inhibition, affects multiple functions of isolated normal human lymphocytes and dendritic cells. Cancer Immunol Res 2(4):351-360

16. Zhao Y, Adjei AA (2014) The clinical development of MEK inhibitors. Nat Rev Clin Oncol 11(7):385-400

17. Zeiser R (2014) Trametinib. Recent Results Cancer Res 201: 241-248

18. Martin-Liberal J, Lagares-Tena L, Larkin J (2014) Prospects for MEK inhibitors for treating cancer. Expert Opin Drug Saf 13(4): 483-495

19. Adjei AA, Cohen RB, Franklin W, Morris C, Wilson D, Molina JR, Hanson LJ, Gore L, Chow L, Leong S, Maloney L, Gordon G, Simmons H, Marlow A, Litwiler K, Brown S, Poch G, Kane K, Haney J, Eckhardt SG (2008) Phase I pharmacokinetic and pharmacodynamic study of the oral, small-molecule mitogen-activated protein kinase kinase 1/2 inhibitor AZD6244 (ARRY-142886) in patients with advanced cancers. J Clin Oncol 26(13):2139-2146

20. Miller CR, Oliver KE, Farley JH (2014) MEK1/2 inhibitors in the treatment of gynecologic malignancies. Gynecol Oncol 133(1):128-137

21. Salama AK, Kim KB (2013) MEK inhibition in the treatment of advanced melanoma. Curr Oncol Rep 15(5):473-482

22. Jain N, Curran E, Iyengar NM, Diaz-Flores E, Kunnavakkam R, Popplewell L, Kirschbaum MH, Karrison T, Erba HP, Green M, Poire X, Koval G, Shannon K, Reddy PL, Joseph L, Atallah EL, Dy P, Thomas SP, Smith SE, Doyle LA, Stadler WM, Larson RA, Stock W, Odenike O (2014) Phase II study of the oral MEK inhibitor selumetinib in advanced acute myelogenous leukemia: a University of Chicago phase II consortium trial. Clin Cancer Res 20(2):490-498

23. Wright CJ, McCormack PL (2013) Trametinib: first global approval. Drugs 73(11):1245-1254

24. Dong Q, Dougan DR, Gong X, Halkowycz P, Jin B, Kanouni T, O'Connell SM, Scorah N, Shi L, Wallace MB, Zhou F (2011) Discovery of TAK-733, a potent and selective MEK allosteric site inhibitor for the treatment of cancer. Bioorg Med Chem Lett 21(5): 1315-1319

25. Micel LN, Tentler JJ, Tan AC, Selby HM, Brunkow KL, Robertson KM, Davis SL, Klauck PJ, Pitts TM, Gangolli E, Fabrey R, O'Connell SM, Vincent PW, Eckhardt SG (2015) Antitumor activity of the MEK inhibitor TAK-733 against melanoma cell lines and patient-derived tumor explants. Mol Cancer Ther 14(2):317-325

26. von Euw E, Atefi M, Attar N, Chu C, Zachariah S, Burgess BL, Mok S, Ng C, Wong DJ, Chmielowski B, Lichter DI, Koya RC, McCannel TA, Izmailova E, Ribas A (2012) Antitumor effects of the investigational selective MEK inhibitor TAK733 against cutaneous and uveal melanoma cell lines. Mol Cancer 11:22

27. Fabrey R, O'Connell SM, Tsuchiya S, Hori A, Vincent PW (2010) TAK-733 is a potent and selective inhibitor of mammalian MEK kinases capable of inducing apoptosis in BRAF driven cells both in vitro and in vivo (Abstract). Cancer Res 70:2518

28. Nakamura A, Arita T, Tsuchiya S, Donelan J, Chouitar J, Carideo E, Galvin K, Okaniwa M, Ishikawa T, Yoshida S (2013) Antitumor activity of the selective pan-RAF inhibitor TAK-632 in BRAF inhibitor-resistant melanoma. Cancer Res 73(23):7043-7055

29. Miyake H, Tsuchiya S, Hoshino T, Hori A, Vincent PW (2010) Antitumor effects of TAK-733, a novel MEK1/2 inhibitor, against mesothelioma cells (Abstract). Cancer Res 70:2516

30. Lieu CH, Tentler JL, Tan AC, Pitts TM, Spreafico A, Selby HM, McPhillips KL, Bagby SM, Eckhardt SG (2012) TAK-733, an investigational novel MEK inhibitor, suppresses colorectal cancer (CRC) tumor growth in biomarker positive patient-derived human tumor explants (Abstract). Eur J Cancer 48:119-120

31. Ishino S, Miyake H, Vincent P, Mori I (2015) Evaluation of the therapeutic efficacy of a MEK inhibitor (TAK-733) using (18)Ffluorodeoxyglucose-positron emission tomography in the human lung xenograft model A549. Ann Nucl Med 29(7):613-620

32. Azijli K, Stelloo E, Peters GJ, Van Den Eertwegh AJ (2014) New developments in the treatment of metastatic melanoma: immune checkpoint inhibitors and targeted therapies. Anticancer Res 34(4):1493-1505

33. Eisenhauer EA, Therasse P, Bogaerts J, Schwartz LH, Sargent D, Ford R, Dancey J, Arbuck S, Gwyther S, Mooney M, Rubinstein L, Shankar L, Dodd L, Kaplan R, Lacombe D, Verweij J (2009) New response evaluation criteria in solid tumours: revised RECIST guideline (version 1.1). Eur J Cancer 45(2):228-247

34. Akinleye A, Furqan M, Mukhi N, Ravella P, Liu D (2013) MEK and the inhibitors: from bench to bedside. J Hematol Oncol 6(27)

35. Kim DW, Patel SP (2014) Profile of selumetinib and its potential in the treatment of melanoma. Onco Targets Ther 7:1631-1639

36. Flaherty KT, Robert C, Hersey P, Nathan P, Garbe C, Milhem M, Demidov LV, Hassel JC, Rutkowski P, Mohr P, Dummer R, Trefzer U, Larkin JM, Utikal J, Dreno B, Nyakas M, Middleton MR, Becker JC, Casey M, Sherman LJ, Wu FS, Ouellet D, Martin AM, Patel K, Schadendorf D (2012) Improved survival with MEK inhibition in BRAF-mutated melanoma. N Engl J Med 367(2):107-114

37. Infante JR, Fecher LA, Falchook GS, Nallapareddy S, Gordon MS, Becerra C, DeMarini DJ, Cox DS, Xu Y, Morris SR, Peddareddigari VG, Le NT, Hart L, Bendell JC, Eckhardt G, Kurzrock R, Flaherty K, Burris HA III, Messersmith WA (2012) Safety, pharmacokinetic, pharmacodynamic, and efficacy data for the oral MEK inhibitor trametinib: a phase 1 dose-escalation trial. Lancet Oncol 13(8): 773-781

38. Falchook GS, Lewis KD, Infante JR, Gordon MS, Vogelzang NJ, DeMarini DJ, Sun P, Moy C, Szabo SA, Roadcap LT, Peddareddigari VG, Lebowitz PF, Le NT, Burris HA III, Messersmith WA, O'Dwyer PJ, Kim KB, Flaherty K, Bendell JC, Gonzalez R, Kurzrock R, Fecher LA (2012) Activity of the oral MEK inhibitor trametinib in patients with advanced melanoma: a phase 1 dose-escalation trial. Lancet Oncol 13(8):782-789

39. Leijen S, Middleton MR, Tresca P, Kraeber-Bodere F, Dieras V, Scheulen ME, Gupta A, Lopez-Valverde V, Xu ZX, Rueger R, Tessier JJ, Shochat E, Blotner S, Naegelen VM, Schellens JH, Eberhardt WE (2012) Phase I dose-escalation study of the safety, pharmacokinetics, and pharmacodynamics of the MEK inhibitor RO4987655 (CH4987655) in patients with advanced solid tumors. Clin Cancer Res 18(17):4794-4805

40. Haura EB, Ricart AD, Larson TG, Stella PJ, Bazhenova L, Miller VA, Cohen RB, Eisenberg PD, Selaru P, Wilner KD, Gadgeel SM (2010) A phase II study of PD-0325901, an oral MEK inhibitor, in previously treated patients with advanced non-small cell lung cancer. Clin Cancer Res 16(8):2450-2457

41. Anforth R, Liu M, Nguyen B, Uribe P, Kefford R, Clements A, Long GV, Fernandez-Penas P (2013) Acneiform eruptions: a common cutaneous toxicity of the MEK inhibitor trametinib. Australas J Dermatol 55(4):250-254

42. Curry JL, Torres-Cabala CA, Kim KB, Tetzlaff MT, Duvic M, Tsai KY, Hong DS, Prieto VG (2014) Dermatologic toxicities to targeted cancer therapy: shared clinical and histologic adverse skin reactions. Int J Dermatol 53(3):376-384

43. Balagula Y, Barth HK, Busam KJ, Lacouture ME, Chapman PB, Myskowski PL (2011) Dermatologic side effects associated with the MEK 1/2 inhibitor selumetinib (AZD6244, ARRY-142886). Investig New Drugs 29(5):1114-1121 
44. Urner-Bloch U, Urner M, Stieger P, Galliker N, Winterton N, Zubel A, Moutouh-de Parseval L, Dummer R, Goldinger SM (2014) Transient MEK inhibitor-associated retinopathy in metastatic melanoma. Ann Oncol 25(7):1437-1441

45. McCannel TA, Chmielowski B, Finn RS, Goldman J, Ribas A, Wainberg ZA, McCannel CA (2014) Bilateral subfoveal neurosensory retinal detachment associated with MEK inhibitor use for metastatic cancer. JAMA Ophthalmol 132(8):1005-1009

46. Adjei AA, LoRusso P, Ribas A, Sosman JA, Pavlick AC, Dy GK, Zhou X, Gangolli EA, Walker RM, Kneissl M, Faucette S, Neuwirth R, Bozon V (2013) Phase I, dose-escalation study of the investigational drug TAK-733, an oral MEK inhibitor, in patients (pts) with advanced solid tumors (Abstract). J Clin Oncol 31:2528

47. Cohen RB, Aamdal S, Nyakas M, Cavallin M, Green D, Learoyd M, Smith I, Kurzrock R (2013) A phase I dose-finding, safety and tolerability study of AZD8330 in patients with advanced malignancies. Eur J Cancer 49(7):1521-1529

48. Honda K, Yamamoto N, Nokihara H, Tamura Y, Asahina H, Yamada Y, Suzuki S, Yamazaki N, Ogita Y, Tamura T (2013)
Phase I and pharmacokinetic/pharmacodynamic study of RO5126766, a first-in-class dual Raf/MEK inhibitor, in Japanese patients with advanced solid tumors. Cancer Chemother Pharmacol 72(3):577-584

49. Long GV, Stroyakovskiy D, Gogas H, Levchenko E, de Braud F, Larkin J, Garbe C, Jouary T, Hauschild A, Grob JJ, Chiarion Sileni V, Lebbe C, Mandala M, Millward M, Arance A, Bondarenko I, Haanen JB, Hansson J, Utikal J, Ferraresi V, Kovalenko N, Mohr P, Probachai V, Schadendorf D, Nathan P, Robert C, Ribas A, DeMarini DJ, Irani JG, Casey M, Ouellet D, Martin AM, Le N, Patel K, Flaherty K (2014) Combined BRAF and MEK inhibition versus BRAF inhibition alone in melanoma. N Engl J Med 371(20): $1877-1888$

50. Robert C, Karaszewska B, Schachter J, Rutkowski P, Mackiewicz A, Stroiakovski D, Lichinitser M, Dummer R, Grange F, Mortier L, Chiarion-Sileni V, Drucis K, Krajsova I, Hauschild A, Lorigan P, Wolter P, Long GV, Flaherty K, Nathan P, Ribas A, Martin AM, Sun P, Crist W, Legos J, Rubin SD, Little SM, Schadendorf D (2015) Improved overall survival in melanoma with combined dabrafenib and trametinib. N Engl J Med 372(1):30-39 